\title{
THE LOGICAL CONTINGENCY OF IDENTITY
}

\author{
HANOCH BEN-YAMI \\ Central European University \\ Original scientific article - Received: 23/03/2018 Accepted: 04/07/2018
}

\begin{abstract}
I show that intuitive and logical considerations do not justify introducing Leibniz's Law of the Indiscernibility of Identicals in more than a limited form, as applying to atomic formulas. Once this is accepted, it follows that Leibniz's Law generalises to all formulas of the first-order Predicate Calculus but not to modal formulas. Among other things, identity turns out to be logically contingent.
\end{abstract}

Keywords: Leibniz's Law, indiscernibility of identicals, identity, necessity, contingency

There is a straightforward formal proof of the necessity of identity, going back at least to Quine $(1953,156)$. It relies on the two derivation rules, necessitation (NEC) and Leibniz's Law of the Indiscernibility of Identicals (LL). NEC enables us to derive $\square$ A ('necessarily A') from any theorem A. Leibniz's Law allows us to substitute identicals in any formula:

$$
\text { Leibniz's Law: } a=b, \mathrm{~A}(a) \vdash \mathrm{A}(b / a)
$$

$\mathrm{A}(b / a)$ is the result of substituting none, some or all occurrences of ' $a$ ' in 'A' by ' $b$ '. One formulation and derivation of the necessity of identity runs as follows:

$\begin{array}{llll}1 & \text { (1) } & a=b & \text { Premise } \\ & (2) & a=a & \text { Axiom (Law of Identity) } \\ & (3) & \square(a=a) & \text { NEC 2 } \\ 1 & (4) & \square(a=b) & \text { LL 1, 3 } \\ & (5) & (a=b) \rightarrow \square(a=b) & \text { Implication Introduction 1, 4 }\end{array}$


The line number is in parentheses; numbers to its left designate the line numbers of the premises, if any, on which the formula in that line depends. As can be seen, the formula in line (5) depends on no premise and is thus a theorem. It says that if $a$ is identical to $b$, then $a$ is necessarily identical to $b$.

Although there is a long tradition of considering this result problematic (see survey and references in (Burgess 2014)), there is currently no generally accepted way of showing the formal considerations uncompelling. In this paper, I try to provide reasons for holding them such. ${ }^{1}$

My basic claim is that Leibniz's Law has been overgeneralised. Suppose $a$ is $b$, and suppose $a$ has a certain property; then $b$, namely $a$, has it. Also, if $a$ stands in any relation, then $b$, namely $a$, stands in that relation. These intuitive claims justify Leibniz's Law as applying to properties and relations. To formalise it in logic, we should allow the substitution of identicals in formulas that express what we intuitively count as properties and relations, namely, one- and many-place predicates. For instance, if $a=b$ and $\mathrm{P}(a)$, then $\mathrm{P}(b)$; or, if $a=b$ and $\mathrm{R}(a, c)$, then $\mathrm{R}(b, c)$. More generally, for any n-place predicate $\mathrm{P}^{\mathrm{n}}$, from $a=b$ and $\mathrm{P}^{\mathrm{n}}(\ldots a \ldots)$ we can infer $\mathrm{P}^{\mathrm{n}}(\ldots b / a \ldots)$ :

$$
a=b, \mathrm{P}^{\mathrm{n}}(\ldots a \ldots) \vdash \mathrm{P}^{\mathrm{n}}(\ldots b / a \ldots)
$$

However, this intuitive justification of Leibniz's Law does not support formalising it as allowing the substitution of identicals in more complex formulas, non-atomic ones, which do not just express properties or relations. It is not that such substitution is prohibited by the intuitive justification, but if it is to be allowed then it should be justified by deriving it from the intuitive version or its formalisation.

This cautionary note is further supported by the fact that, with some sentences of more complex kinds, the applicability of Leibniz's Law is far from certain. Although Mr Utterson knows he's an old friend of Dr Henry Jekyll, does he also know he's an old friend of Mr Edward Hyde? Even if he does, this should be shown. Namely, we cannot assume that Leibniz's Law applies to all sentences but we need to derive its applicability from its limited, basic form, applied only to atomic sentences.

\footnotetext{
${ }^{1}$ A referee for this journal drew my attention to (Garson 2013: §12.3), where similar ideas to some of those developed below are to be found. Garson, however, justifies his views by claiming that substitutivity does not generally hold in modal contexts, while I attempt to derive this.
} 
In fact, introducing Leibniz's Law into logic in its limited form does entail its application to all formulas of the standard version of the Predicate Calculus. I shall show this by providing an outline of an inductive proof of this claim. (In order not to make this paper too technical, some of the results are only stated; in all these cases establishing them shouldn't be difficult for anyone familiar with the relevant systems.) The proof is by induction on formula complexity (see Mendelson 1996, 96). Let us assume our calculus contains only three logical constants: $\neg, \wedge$ and $\forall$.

First, LL applies, by definition, to all atomic formulas.

Assume LL applies to any formulas $\mathrm{A}(a)$ and $\mathrm{B}(a)$, and let us show that it applies to $\mathrm{A}(a) \wedge \mathrm{B}(a)$. That is, we have to show that if $a=b$ and $\mathrm{A}(a) \wedge \mathrm{B}(a)$, then $(\mathrm{A}(a) \wedge \mathrm{B}(a))(b / a)$, namely $\mathrm{A}(b / a) \wedge \mathrm{B}(b / a)$. The proof proceeds as follows:

$\begin{array}{llll}1 & (1) & \mathrm{A}(a) \wedge \mathrm{B}(a) & \text { Premise } \\ 2 & (2) & a=b & \text { Premise } \\ 1 & (3) & \mathrm{A}(a) & \text { Conjunction Elimination 1 } \\ 1 & (4) & \mathrm{B}(a) & \text { Conjunction Elimination 1 } \\ 1,2 & (5) & \mathrm{A}(b / a) & \text { LL was assumed to apply to } \mathrm{A}(a) \\ 1,2 & (6) & \mathrm{B}(b / a) & \text { LL was assumed to apply to } \mathrm{B}(a) \\ 1,2 & (7) & \mathrm{A}(b / a) \wedge \mathrm{B}(b / a) & \text { Conjunction Introduction 5, 6 }\end{array}$

I skip the proof for $\neg$. We now prove the theorem for $\forall \mathrm{xA}(\mathrm{x}, a)$, assuming that LL applies to all formulas $\mathrm{A}(c / \mathrm{x}, a)$, in which a constant $c$ replaced all occurrences of $\mathrm{x}$ in $\mathrm{A}(\mathrm{x}, a)$. We have to show that if $a=b$ and $\forall \mathrm{xA}(\mathrm{x}, a)$, then $\forall \mathrm{xA}(\mathrm{x}, b / a)$.

$\begin{array}{llll}1 & \text { (1) } & \forall \mathrm{xA}(\mathrm{x}, a) & \text { Premise } \\ 2 & \text { (2) } & a=b & \text { Premise } \\ 1 & \text { (3) } & \mathrm{A}(c / \mathrm{x}, a) & \begin{array}{l}\text { Universal Elimination } 1 \text { (' } c \text { ' shouldn't } \\ \text { occur in (1) or (2)) }\end{array} \\ 1,2 & \text { (4) } & \mathrm{A}(c / \mathrm{x}, b / a) & \text { LL was assumed to apply to } \mathrm{A}(\mathrm{c} / \mathrm{x}, a) \\ 1,2 & \text { (5) } & \forall \mathrm{xA}(\mathrm{x}, b / a) & \text { Universal Introduction } 4\end{array}$

Accordingly, introducing Leibniz's Law in its limited form, in which it applies only to atomic formulas, suffices for its applicability to all formulas of the standard version of the Predicate Calculus. 
Since what can be proved should not be given the status of a principle, the proof system does not justify introducing Leibniz's Law in more than its limited form.

Semantic considerations also show that if Leibniz's Law is introduced as a principle in its limited form then it generalises to all formulas of the standard version of the Predicate Calculus. Namely, if the semantics respects Leibniz's Law in its limited form, as well as the standard truthvalue rules for connectives and quantifiers, the applicability in the semantics of Leibniz's Law to all formulas of the standard version of the Predicate Calculus follows. In the semantics, Leibniz's Law in its limited form means that if $\mathrm{V}$ is a function that specifies the truth-values of formulas in a model, and $\mathrm{V}[a=b]=\mathrm{T}$, then for every $\mathrm{n}$-place predicate $\mathrm{P}^{\mathrm{n}}$, $\mathrm{V}\left[\mathrm{P}^{\mathrm{n}}(\ldots a \ldots)\right]=\mathrm{V}\left[\mathrm{P}^{\mathrm{n}}(\ldots b / a \ldots)\right]$.

The proof of this claim is again by induction on formula complexity. Here I shall show it only for the case of negation. Namely, we assume that $\mathrm{V}[a=b]=\mathrm{T}$ and that $\mathrm{V}[\mathrm{A}(a)]=\mathrm{V}[\mathrm{A}(b / a)]$, and show that $\mathrm{V}[\neg \mathrm{A}(a)]=\mathrm{V}[\neg \mathrm{A}(b / a)]$. Since we assume that the semantics respects the truth-value rules for connectives, for any formula $\varphi, \mathrm{V}[\neg \varphi]=\mathrm{T}$ if $\mathrm{V}[\varphi]=\mathrm{F}$ and $\mathrm{V}[\neg \varphi]=\mathrm{F}$ if $\mathrm{V}[\varphi]=\mathrm{T}$. Accordingly, $\mathrm{V}[\neg \mathrm{A}(b / a)]=\mathrm{T}(\mathrm{F})$ just in case $\mathrm{V}[\mathrm{A}(b / a)]=\mathrm{F}(\mathrm{T})$, which holds, according to our assumption, if and only if $\mathrm{V}[\mathrm{A}(a)]=\mathrm{F}(\mathrm{T})$, but then $\mathrm{V}[\neg \mathrm{A}(a)]=\mathrm{T}(\mathrm{F})$, which is what we had to prove.

Again, since it is unjustified to introduce a rule as a principle if it follows from another, less general principle, the semantics also does not justify conferring the status of a principle on Leibniz's Law in its general form.

We see that both intuitively and formally, and the latter for both provability and semantic considerations, it is unjustified to introduce Leibniz's Law in more than its limited form, namely as applying only to atomic formulas.

This revision of Leibniz's Law has significant consequences when we move to modal logic. Given the standard laws of inference, substitutivity in modal contexts cannot be proved, even for a system as strong as S5. Namely, generally

$$
a=b, \square \mathrm{A}(a) \Vdash \square \mathrm{A}(b / a)
$$

(I do not provide here a proof of this improvability; it can be proved from the soundness of the modal systems.) This renders invalid the move from line (3) to line (4) in the proof that opened this paper, namely from $\square(a=a)$ to $\square(a=b)$. No other proof of the conclusion can be provided. 
The case is similar with the semantics of modal logic. Namely, we can adopt as a semantic principle Leibniz's Law in its limited form without identity being necessary. For instance, in possible-worlds semantics, if ' $a$ ' and ' $b$ ' designate one object $\alpha$ at world $\mathrm{w}_{1}$ but two different objects, $\alpha$ and $\beta$, at world $\mathrm{w}_{2}$ which is accessible from $\mathrm{w}_{1}$, then although ' $a=b$ ' is true at $\mathrm{W}_{1}$, ' $\square(a=b)$ ' is false at it (because ' $a=b$ ' is false at $\left.\mathrm{w}_{2}\right)$; at the same time, if ' $\mathrm{P}^{\mathrm{n}}(\ldots a \ldots)$ ' is true at $\mathrm{w}_{1}$, then so is ' $\mathrm{P}^{\mathrm{n}}(\ldots b / a \ldots)$ '. We thus have, with ' $a=a$ ' as 'A $(a)$ ' and ' $a=b$ ' as ' $\mathrm{A}(b / a)$ ':

$$
\begin{aligned}
& a=b, \mathrm{P}^{\mathrm{n}}(\ldots a \ldots) \vDash \mathrm{P}^{\mathrm{n}}(\ldots b / a \ldots) \\
& a=b, \square \mathrm{A}(a) \not \neq \square \mathrm{A}(b / a)
\end{aligned}
$$

It can be shown that if we limit Leibniz's Law in the ways described above to atomic formulas in both the proof systems and the semantics of the common formal systems, these systems remain sound and complete. ${ }^{2}$ Limiting Leibniz's Law in this way therefore does not have any undesirable metalogical consequences.

Of course, one can force a semantics that makes identity necessary, which is the semantics that has standardly been used. Namely, one can stipulate that if ' $a$ ' and ' $b$ ' designate $\alpha$ at world $\mathrm{w}_{1}$ then both designate $\alpha$ at any world accessible from $\mathrm{w}_{1}$. The point is that formal considerations do not justify such a stipulation.

The necessity of distinctness, proved by using the necessity of identity (see, for instance, (Burgess 2011,319)), is also invalid once we limit Leibniz's Law to n-place predicates.

To conclude, if we adopt as a logical principle the form of Leibniz's Law which I find more intuitive and logically justified, namely, the form applicable only to properties and relations, the necessity of identity does not follow from formal considerations, and this without having any undesirable formal consequences. One might try to provide other reasons for this alleged necessity, as metaphysicians have tried to do (whether successfully or not is not the subject of this paper). If successful, necessary identity can then be represented in our logic systems. Yet from a logical point of view, identity is better left contingent. ${ }^{3}$

\footnotetext{
${ }^{2}$ For some discussion of modal systems with contingent identity, see Hughes and Cresswell (1996: 330ff).

${ }^{3}$ Thanks to Daniel Kodaj, Ran Lanzet, Edi Pavlovic and an anonymous referee for comments on an earlier version of this paper.
} 


\section{REFERENCES}

Burgess, J. 2011. The logic of necessity. In The Bloomsbury Companion to Philosophical Logic, eds. L. Horsten and R. Pettigrew, 299323. London: Bloomsbury Academic.

Burgess, J. 2014. On a derivation of the necessity of identity. Synthese 191: $1567-1585$.

Garson, J. W. 2013. Modal Logic for Philosophers. $2^{\text {nd }}$ edition. Cambridge: Cambridge University Press.

Hughes, G. E. and M. J. Cresswell. 1996. A New Introduction to Modal Logic. London: Routledge.

Mendelson, E. 1996. Introduction to Mathematical Logic. $4^{\text {th }}$ edition. London: Chapman \& Hall.

Quine, W. v. 1953. Reference and modality. In his From a Logical Point of View. $2^{\text {nd }}$ edition 1963, 139-159. Cambridge, MA: Harvard University Press. 